\title{
Diagnostic accuracy of clinical examination for detection of non-cephalic presentation in late pregnancy: cross sectional analytic study
}

Natasha Nassar, Christine L Roberts, Carolyn A Cameron, Emily C Olive

\begin{abstract}
Objective To examine the diagnostic accuracy of clinical examination to determine fetal presentation in late pregnancy. Design Cross sectional analytic study with index test of clinical examination and reference standard of ultrasonography.

Setting Antenatal clinic in tertiary obstetric hospital in Sydney, Australia.

Participants 1633 women with a singleton pregnancy between 35 and 37 weeks' gestation attending antenatal clinics. Intervention Fetal presentation assessed by clinical examination during routine antenatal care, followed by ultrasonography to confirm the diagnosis.

Main outcome measures Sensitivity, specificity, and positive and negative predictive values of clinical examination compared with ultrasonography. Diagnostic rates by maternal characteristics.

Results Ultrasonography identified non-cephalic presentation in $130(8 \%)$ women, comprising 103 (6.3\%) with breech and 27 $(1.7 \%)$ with transverse or oblique lie. Sensitivity of clinical examination for detecting non-cephalic presentation was $70 \%$ (95\% confidence interval $62 \%$ to $78 \%$ ) and specificity was $95 \%$ (94\% to $96 \%$ ). The positive predictive value and negative predictive value were $55 \%$ and $97 \%$, respectively.

Conclusions Clinical examination is not sensitive enough for detection and timely management of non-cephalic presentation.
\end{abstract}

\section{Introduction}

Antenatal detection of non-cephalic presentation-comprising breech presentation and transverse or oblique lie-in late pregnancy is important for timely management and clinical decision making. For breech presentation, women and their clinicians must decide whether to try external cephalic version to increase the likelihood of vaginal birth or plan a caesarean section, with optimal gestation being 37 and 39 weeks, respectively. ${ }^{1}$ Diagnosis of non-cephalic presentation after the onset of labour is associated with increased maternal and infant morbidity and mortality. ${ }^{2}$

Fetal presentation is generally assessed by palpating the abdomen (clinical examination), though we do not know the accuracy of this in late pregnancy. ${ }^{3-6}$ We conducted a cross sectional analytic study to compare clinical examination with the reference standard of ultrasonography.

\section{Methods \\ Patients, setting, and data collection}

We carried out the study at an antenatal clinic in a tertiary obstetric hospital between September 2003 and December 2004. Women with a singleton pregnancy at 35-37 weeks' gestation were eligible. A midwife, resident, registrar, or obstetrician, all of whom were aware of the study, provided routine antenatal care. All eligible women underwent clinical examination to assess fetal presentation. Subsequently, those who consented to participate underwent ultrasonography to confirm the diagnosis. The ultrasound examination was conducted with a portable hand held machine following a standard protocol. The operators were blinded to the result of the clinical examination until after the ultrasonography.

We collected data from the antenatal record and recorded it on a standard data abstraction form. We assessed the accuracy of clinical examination in diagnosing fetal presentation by calculating sensitivity, specificity, and positive and negative predictive values.

\section{Sample size and analysis}

To determine a sensitivity of $75 \%$ (with a $95 \%$ confidence interval plus or minus $10 \%$ ) we required 100 women with a breech presentation. As $6-8 \%$ of singleton pregnancies are breech at 35-37 weeks' gestation, ${ }^{7}$ we needed between 1250 and 1700 women to gain a sample of 100 with a breech presentation. We investigated predictive factors associated with correct diagnosis of fetal presentation using contingency tests and used sensitivity analyses to examine specific accuracy rates by maternal characteristics. $\mathrm{P}<0.05$ was considered significant and analyses were conducted with SAS version 8.2 (SAS Institute, Cary, NC).

\section{Results}

Of the 1707 eligible women approached, 65 women refused to take part because of lack of time or concern about having had too many ultrasound examinations during their pregnancy, and nine women were excluded owing to missing data. The average age of the 1633 participating women was 31 years (SD 5.4); $55 \%$ were nulliparous; $31 \%$ were overweight or obese; and $61 \%$ were white. Over 60 care providers participated, with $55 \%$ of examinations performed by residents or registrars, $28 \%$ by midwives, and $17 \%$ by obstetricians.

Ultrasonography identified non-cephalic presentation in 130 (8\%) women, comprising $103(6.3 \%)$ with breech and $27(1.7 \%)$ with transverse or oblique lie. The sensitivity of clinical examination for identifying non-cephalic presentation was $70 \%$ and spe- 
Sensitivity and specificity (as \%) of clinical examination for detecting fetal presentation

\begin{tabular}{|c|c|c|c|c|}
\hline \multirow{2}{*}{ Characteristic } & \multicolumn{2}{|c|}{ Non-cephalic, correctly diagnosed } & \multicolumn{2}{|c|}{ Cephalic, correctly diagnosed } \\
\hline & No of cases & Sensitivity $(95 \% \mathrm{CI})$ & No of cases & Specificity (95\% CI) \\
\hline Overall & $91 / 130$ & 70 (62 to 78) & $1429 / 1503$ & 95 (94 to 96) \\
\hline \multicolumn{5}{|l|}{ Maternal age (years): } \\
\hline$<35$ & $55 / 84$ & 65 (64 to 66) & $1100 / 1158$ & 95 (94 to 96) \\
\hline$\geq 35$ & $34 / 44$ & 77 (75 to 79$)$ & $319 / 336$ & 95 (94 to 96$)$ \\
\hline \multicolumn{5}{|l|}{ Gestational age (weeks): } \\
\hline $34-35$ & $32 / 41$ & 78 (76 to 80$)$ & $316 / 343$ & 92 (91 to 93) \\
\hline 36 & $29 / 44$ & 66 (64 to 68) & $492 / 512$ & 96 (95 to 97) \\
\hline $37-38$ & $28 / 43$ & 65 (63 to 67) & $615 / 641$ & 96 (95 to 97) \\
\hline \multicolumn{5}{|l|}{ Parity: } \\
\hline Nulliparous & $49 / 73$ & 67 (65 to 68 ) & $779 / 820$ & 95 (94 to 96$)$ \\
\hline Multiparous & $42 / 57$ & 74 (72 to 75$)$ & $643 / 677$ & 95 (94 to 96) \\
\hline \multicolumn{5}{|l|}{ Body mass index: } \\
\hline Thin & $9 / 13$ & 69 (62 to 76$)$ & $109 / 115$ & 95 (94 to 96$)$ \\
\hline Normal weight & $49 / 67$ & 73 (72 to 74$)$ & $862 / 898$ & 96 (95 to 97) \\
\hline Overweight & $19 / 28$ & 68 (65 to 71) & $234 / 241$ & 97 (96 to 98) \\
\hline \multicolumn{5}{|l|}{ Country of birth: } \\
\hline Australia/New Zealand/Europe & $52 / 72$ & 72 (71 to 73 ) & $855 / 900$ & 95 (94 to 96$)$ \\
\hline Asia & $18 / 39$ & 46 (67 to 72$)$ & $407 / 424$ & 96 (95 to 97) \\
\hline Other & $8 / 11$ & 69 (37 to 54) & $144 / 152$ & 95 (94 to 96) \\
\hline
\end{tabular}

Numbers may not add up to totals because of missing data.

cificity was $95 \%$ (table). A similar rate of sensitivity was found for breech presentation $(70 \%, 61 \%$ to $78 \%)$. The positive and negative predictive values were $55 \%$ and $97 \%$, respectively.

The sensitivity of clinical examination for determining non-cephalic presentation was not associated with any particular maternal characteristics, but there was a trend of increasing sensitivity for women with a previous pregnancy (multiparous) and lower body mass index (table). The proportion of women in whom cephalic presentation was correctly diagnosed (specificity) was significantly greater with increasing gestational age and decreasing body mass index $(\mathrm{P}<0.05)$ (table).

\section{Discussion}

In this large study in a general maternity population we found that clinical examination was, generally, not sensitive enough to accurately diagnose fetal presentation in late pregnancy. Although clinical examination increased the probability of diagnosis from $8 \%$ (prior probability or prevalence) to $55 \%$ (posterior probability or positive predictive value), ${ }^{8}$ only $70 \%$ of non-cephalic presentations were detected. If we apply our findings to a general maternity population of 1000 women, clinical examination would identify 101 women as having a non-cephalic presentation but in only 56 would this be correct; and 24 women with non-cephalic presentation would be missed altogether.

\section{Strengths and limitations of the study}

We included a large unselected sample and used appropriate timing of the clinical examination relevant for management of non-cephalic presentation in late pregnancy. Previous reports of the sensitivity of clinical examination for detecting non-cephalic presentation have ranged from 28-88\%. These studies were small, underpowered, and included selected high risk pregnancies and low gestational ages (range 20-42 weeks). ${ }^{3-6}$ Our observed prevalence of non-cephalic presentation was consistent with rates found in longitudinal studies of fetal presentation, ${ }^{9}$ suggesting that our findings may be applied in other obstetric settings.
We did not collect information on individual clinicians and were unable to ascertain whether particular individuals may have biased results. As all examiners were aware of the study and assessments were recorded and verified, we assumed that assessors would be vigilant. Nevertheless, it is possible that some clinicians may not have been as attentive because diagnoses were going to be checked with ultrasonography.

\section{Room for improvement}

Introduction of routine ultrasonography to assess fetal presentation in late pregnancy would improve diagnostic accuracy. However, costs, resource availability, and feasibility need to be considered, as well as the potential deskilling of care providers in performing clinical examination. A cost effectiveness analysis would be necessary before implementation and change in clinical obstetric practice. However, lower rates of accuracy found among overweight or obese women suggest that formal ultrasonography in late pregnancy for these women is required.

Clinical examination to assess fetal presentation is a relatively simple procedure and, with ongoing diligence and regular audit and feedback, accuracy may be increased. Variability in accuracy rates by examiner and level of experience also suggest there is room for improvement by all pregnancy care providers. ${ }^{3510}$

We thank the staff and the women at Royal Prince Alfred Women and Babies Hospital who participated in the study for their time and cooperation. We particularly thank Hala Phipps, Sarah Charlton, and Julie

\section{What is already known on this topic}

There is limited information about the accuracy of clinical examination for detection of fetal presentation in late pregnancy

\section{What this study adds}

Compared with ultrasonography, the sensitivity of clinical examination is inadequate for detection and timely management of non-cephalic presentation 
Bedford for recruiting women and performing the ultrasound examinations.

Contributors: All authors contributed to the study design and protocol development. NN and CLR supervised the conduct of the study, and ECO conducted staff training. NN performed the data analysis and is guarantor. All authors contributed to the interpretation of findings and writing and review of the manuscript.

Funding: Australian National Health and Medical Research Council (NHMRC) project grant (211051). NN was funded by an Australian NHMRC Public Health Postgraduate Research Scholarship. CLR was funded by an Australian NHMRC Public Health Practitioner Fellowship.

Competing interests: None declared.

Ethical approval: Approved by the Central Sydney Area Health Service Research Ethics Committee (Protocol number: X03-0185).

1 Hofmeyr GJ, Kulier R. External cephalic version for breech presentation at term. Cochrane Database Syst Rev 2000;(2):CD000083.

2 Waterstone M, Bewley S, Wolfe C. Incidence and predictors of severe obstetric morbidity: case-control study. BMJ 2001;322:1089-93.

3 Watson WJ, Welter S, Day D. Antepartum identification of breech presentation.J Reprod Med 2004;49:294-6.

4 Thorp JM Jr, Jenkins T, Watson W. Utility of Leopold maneuvers in screening for malpresentation. Obstet Gynecol 1991;78:394-6.
5 McFarlin BL, Engstrom JL, Sampson MB, Cattledge F. Concurrent validity of Leopold's maneuvers in determining fetal presentation and position. $J$ Nurse Midwifery 1985;30:280-4.

6 Lydon-Rochelle M, Albers L, Gorwoda J, Craig E, Qualls C. Accuracy of Leopold maneuvers in screening for malpresentation: a prospective study. Birth 1993;20:132-5.

7 Roberts CL, Nassar N, Raynes-Greenow CH, Peat B. Update on the management of term breech deliveries in NSW, Australia. Aust N Z J Obstet Gynaecol 2003:43:173.

8 Altman DG, Bland JM. Diagnostic tests 2: predictive values. BMJ 1994;309:102.

9 Scheer K, Nubar J. Variation of fetal presentation with gestational age. Am J Obstet Gynecol 1976;125:269-70.

10 Nassar N. Breech presentation: facilitating informed decision-making [dissertation]. Sydney, NSW: University of Sydney, 2005.

(Accepted 29 June 2006)

doi 10.1136/bmj.38919.681563.4F

Centre for Perinatal Health Services Research, School of Public Health, University of Sydney NSW 2006, Australia

Natasha Nassar research associate

Christine L Roberts research director

Carolyn A Cameron research associate

Emily C Olive research fellow in obstetrics

Correspondence to N Nassar natashan@ichr.uwa.edu.au 